\section{Santos FM'}

Rodrigues RGS"

Trindade-Filho EM"'I

Faculdade de Fisioterapia. Universidade de Ciências da Saúde de Alagoas (UNCISAL). Maceió, AL, Brasil

" Laboratório de Instrumentação e Acústica. UNCISAL. Maceió, AL, Brasil

III Departamento de Fisiologia. UNCISAL. Maceió, AL, Brasil

Correspondência | Correspondence: Euclides Maurício Trindade Filho

Departamento de Fisiologia

R. Jorge de Lima, 113

Trapiche da Barra

57010-382 Maceió, AL, Brasil

E-mail: emtf@fapeal.br

\section{Exercício físico versus} programa de exercício pela eletroestimulação com aparelhos de uso doméstico

\section{Physical exercise versus exercise program using electrical stimulation devices for home use}

\section{RESUMO}

OBJETIVO: Avaliar os efeitos da eletroestimulação por aparelhos de uso doméstico sobre o condicionamento neuromuscular.

MÉTODOS: A amostra foi composta por 20 mulheres voluntárias, sedentárias, destras, com idades entre 18 a 25 anos em Maceió, estado de Alagoas, em 2006. As mulheres foram divididas aleatoriamente em dois grupos: as do grupo $\mathrm{A}$ foram submetidas a eletroestimulação passiva com aparelhos comerciais e as do grupo $\mathrm{B}$, a exercício físico com resistência. O programa de treinamento dos grupos totalizou 16 sessões em dois meses, com duas sessões semanais. As comparações do peso corporal, da cirtometria, fleximetria, e da força muscular antes e após os exercícios, foram utilizadas utilizando-se o teste T pareado. Nas comparações entre os grupos A e B, foi utilizado o teste $t$ de Student. O nível de significância adotado foi de $5 \%$.

RESULTADOS: A comparação da força muscular medida de forma subjetiva antes e após cada um dos procedimentos, mostrou que ocorreu aumento da força em ambos os grupos. Foram observados aumentos significantes na massa e na força muscular apenas nos indivíduos que realizaram exercício voluntário. $\mathrm{O}$ exercício físico resistido de flexo-extensão dos joelhos foi efetivo em aumentar massa e força muscular, ao contrário das sessões de eletroestimulação com correntes de freqüência de pulsos de $87 \mathrm{~Hz}$, que não tiveram o mesmo efeito.

CONCLUSÕES: Os resultados encontrados mostraram que os aparelhos de eletroestimulação para ganho passivo de condicionamento físico comercializados são menos eficientes do que a prática de exercício físico voluntário.

DESCRITORES: Esforço físico, fisiologia. Força muscular. Estimulação elétrica. Terapia passiva contínua de movimento. 


\begin{abstract}
OBJECTIVE: To evaluate the effects of electrical muscle stimulation with devices for home use on neuromuscular conditioning.

METHODS: The study sample comprised 20 sedentary, right-handed, voluntary women aged from 18 to 25 years in the city of Maceió, Northeastern Brazil, in 2006. Subjects were randomly divided into two groups: group A included women who underwent muscle stimulation using commercial electrical devices; group B included those women who performed physical activities with loads. The training program for both groups consisted of two weekly sessions for two months, in a total of 16 sessions. Comparisons of body weight, cirtometry, fleximetry, and muscle strength before and after exercise were determined using the paired t-test. For the comparisons between both groups, Student's t-test was used and a 5\% significance level was adopted.
\end{abstract}

RESULTS: Muscle strength subjectively assessed before and after each intervention was increased in both groups. Significant increases in muscle mass and strength were seen only in those subjects who performed voluntary physical activity. Resisted knee flexion and extension exercises effectively increased muscle mass and strength when compared to electrical stimulation at $87 \mathrm{~Hz}$ which did not produce a similar effect.

CONCLUSIONS: The study results showed that electrical stimulation devices for passive physical exercising commercially available are less effective than voluntary physical exercise.

KEY WORDS: Exertion, physiology. Muscle strength. Electric stimulation. Motion therapy, continuous passive.

\section{INTRODUÇÃO}

A atividade física regular é reconhecida por seus efeitos saudáveis nos praticantes. É possível relacioná-la a alterações positivas para combater ou prevenir o aparecimento de diversas doenças, tais como: doenças cardiovasculares, obesidade, diabetes, osteoporose, entre outras. A falta de atividade regular contribui para o processo de cardiopatia ${ }^{8}$ e por esta razão, o sedentarismo aparece como fator de risco para essas doenças. ${ }^{6}$

Muitos idosos experimentam a dificuldade em executar atividades da vida diária porque a força gerada de seus músculos é reduzida. A força muscular declina em aproximadamente $15 \%$ na sexta e sétima décadas de vida e por $30 \%$ nas seguintes. Os músculos mais fracos de uma pessoa idosa podem combinar o problema dos ossos frágeis, aumentando o risco de uma queda e conseqüentemente, fratura.

Recentemente, o exercício físico regular vem sendo substituído por sessões com aparelhos de estimulação elétrica intitulados "emagrecedores", "fortalecedores de músculos" e "fontes de um condicionamento físico ideal".
$\mathrm{Na}$ estimulação elétrica, a atividade fica restrita ao músculo estimulado, sendo menos influenciado pelas outras alterações que podem ocorrer no corpo durante o exercício. A estimulação elétrica superposta sobrepassa os mecanismos normais de controle neuronal. ${ }^{\mathrm{a}}$ Aplicada de forma adequada, a eletroterapia é segura e eficiente para proporcionar padrões de exercícios quando os pacientes são incapazes de realizá-los devido à dor, restrições na amplitude de movimento, ou outras disfunções do sistema neuromuscular. ${ }^{4}$ Mesmo em indivíduos saudáveis, a estimulação elétrica tem se mostrado benéfica quanto a ganhos de força muscular, com a ressalva de que sejam considerados critérios e profissionalismo na aplicabilidade da eletroestimulação (Piazzi et $\mathrm{al}^{7}$ ).

Aparelhos eletroestimuladores vendidos para uso doméstico têm prometido resultados musculares. Assim, o presente estudo teve por objetivo avaliar os efeitos da eletroestimulação com o uso desses aparelhos populares, seus reais efeitos sobre o condicionamento neuromuscular e a possibilidade do surgimento de alterações deletérias.

a Angeli G. Comparação entre as variáveis metabólicas, hemodinâmicas e bioquímicas entre a eletro-estimulação e o exercício de resistência localizada dos músculos abdominais [dissertação de mestrado]. São Paulo: Universidade Federal de São Paulo; 2003. 


\section{MÉTODOS}

O estudo foi realizado em Maceió, estado de Alagoas, durante o primeiro semestre de 2006.

Para o cálculo do tamanho da amostra foi utilizada a variável cirtometria considerando-se uma diferença estimada de 2, erro-padrão de 1,5 , o poder do teste de $85 \%$ e o valor de $\alpha 5 \%$. O tamanho calculado da amostra foi de 20 mulheres. Foram excluídos da amostra indivíduos portadores de lesão ou patologia em joelhos, que tivessem exercido atividade física regular há menos de três meses, e que fizessem uso regular de medicamentos, tais quais diuréticos, corticóides e outros.

Assim, a população estudada foi constituída de 20 estudantes do sexo feminino, sedentárias, com idade de 18 a 25 anos que responderam a anúncios para participar da pesquisa, divulgados na universidade. A amostra foi dividida aleatoriamente nos grupos A e B. $\mathrm{O}$ grupo A foi formado por indivíduos que receberam eletroestimulação com aparelho comercializado nas televendas, o qual não possuía referência além da operacional avaliada pelo Laboratório de Instrumentação e Acústica. O grupo B foi formado por indivíduos que foram submetidos a exercícios físicos de contração concêntrica e excêntrica dos quadríceps com caneleiras de pesos variados.

Uma vez exposto seu circuito interno, o aparelho de eletroestimulação apresentou circuito oscilador resistivo indutivo capacitivo RLC e portas lógicas. Sua voltagem apresentava picos de 132 volts, com duração de 0,3 segundos, caindo rapidamente para um patamar de 25 volts, e posteriormente sendo amortecido. Este ciclo tinha duração de $11,5 \mathrm{~ms}$. O conjunto desses pulsos (trem de pulsos) com duração de 2,04 segundos era aplicado entre intervalos de 3,04 segundos, durante o tempo de operação do aparelho. Em síntese, o amortecimento caracterizava-se por freqüência de $12,5 \mathrm{kHz}$, e o trem de pulso por uma freqüência de $87 \mathrm{~Hz}$; segundo análise realizada com osciloscópio da marca Tektronix (MOD 465). A intensidade da corrente elétrica do aparelho era de 1,45 a 2,79 mA, e foi medida com o multímetro de marca Icel (MD-6110).

Os indivíduos de ambos os grupos foram orientados a não realizar atividades físicas regulares e a manter o peso corporal, evitando dietas no decurso da pesquisa. Depois de definidos os grupos da pesquisa, cada indivíduo foi submetido à avaliação dos seguintes parâmetros: cirtometria da coxa direita e esquerda, fleximetria dos joelhos e força muscular máxima do quadríceps.

Para a cirtometria das coxas direita e esquerda, foram realizadas medidas a sete, 14,21 e $30 \mathrm{~cm}$ acima da margem inferior da patela, utilizando-se de fita métrica com escala em centímetros. A fleximetria da articulação dos joelhos direito e esquerdo foi realizada com o Fleximeter (Instituto Code de Pesquisa/ Londrina-PR), seguindo orientações do manual de instrução.
A força muscular máxima subjetiva foi avaliada pela escala De Lorm. ${ }^{1}$ Dessa forma o indivíduo foi instruído a fazer dez repetições de flexo-extensão do joelho direito com velocidade lenta e constante, sem realizar compensação com os membros superiores ou com os quadris. Estes movimentos foram executados com carga inicial de $3 \mathrm{~kg}$. Esta carga era aumentada progressivamente em $1 \mathrm{~kg}$ de acordo com a capacidade de cada indivíduo realizar o movimento sem desconforto. O limite máximo do peso a ser erguido pelo sujeito foi considerado aquele em que surgisse dor ao movimento ou limitação da amplitude de movimento articular. Foi estipulado um intervalo de descanso de um minuto entre cada carga testada. A carga de resistência para o treino era equivalente a $80 \%$ da resistência máxima avaliada.

A força muscular objetiva dos quadríceps foi avaliada com membro inferior direito e esquerdo em conjunto, utilizando um dinamômetro analógico (Cescom) cuja escala é dada em quilogramas-força (kgf). Cada sujeito foi orientado a ficar a uma distância de $10 \mathrm{~cm}$ da alavanca manual do dinamômetro, e realizar semiflexão de $45^{\circ}$ de quadris e joelhos. Em seguida, o voluntário foi solicitado a realizar extensão vigorosa dos joelhos acompanhada de extensão do tronco ao levantar a alavanca de força do dinamômetro. Foi considerada a força muscular máxima aquela de maior valor após duas aferições.

Os indivíduos dos grupos A e B realizaram duas sessões semanais, em dias alternados, por dois meses.

Os sujeitos do grupo A foram submetidos à eletroestimulação com intensidade suficiente para provocar uma contração muscular visível, considerando o limiar álgico individual. A estimulação tinha duração de 15 min por sessão, respeitando as orientações contidas no manual de instruções do aparelho quanto à colocação dos eletrodos, a fim de estimular o músculo quadríceps.

Os sujeitos do grupo B realizaram três séries de 15 repetições de exercícios de flexo-extensão de joelhos partindo da posição sentada, com quadris e joelhos flexionados a 90 graus, e mãos apoiadas nas coxas para evitar compensações. As séries de exercícios eram intercaladas por um minuto de descanso. Os indivíduos de ambos os grupos foram orientados a realizar o auto-alongamento dos quadríceps duas vezes antes do exercício e duas vezes após, mantendo o alongamento por 20 segundos para cada coxa.

Ao final de dois meses - 16 sessões de exercícios - os indivíduos dos grupos A e B foram submetidos à reavaliação da cirtometria, e avaliação da força muscular máxima do quadríceps, com a finalidade de avaliar possíveis alterações nas medidas observadas anteriormente. 
Para as comparações do peso corporal, da cirtometria, fleximetria, e da força muscular antes e após os exercícios, foi utilizado o teste T Pareado. Nas comparações entre os grupos A e B, foi utilizado o teste t de Student. O nível de significância adotado foi de 5\%. Foi utilizado o software SPSS 13 para realizar as análises estatísticas.

O projeto foi aprovado pela Comissão de Ética da Universidade de Ciências da Saúde de Alagoas.

\section{RESULTADOS}

Ao comparar os dois grupos, as médias de idade foram de 21,9 anos no grupo A e 21,6 anos no grupo B.

Nos indivíduos do grupo A, a força muscular dos quadríceps medida de forma objetiva com o dinamômetro, teve média inicial igual a 47,4 $\mathrm{kgf}$ (IC 95\%: $41,85 ; 52,94)$ e ao fim do treinamento a média foi de 48,9 kgf (IC 95\%: 43,99;53,80) (Figura 1). Isso mostra que não houve diferença significante da força nos indivíduos submetidos à eletroestimulação. $\mathrm{Na}$ avaliação da força muscular do quadríceps pela escala De Lorm, pôde-se constatar aumento significante $(p=0,005)$ nos indivíduos do grupo A ao comparar as medidas finais $(12,35 \mathrm{kgf}$; IC 95\%: 10,05; 14,65) às iniciais $(11,15 \mathrm{kgf}$; IC 95\%: 8,77; 13,42;). As medidas de cirtometria não apresentaram alterações significantes ao longo dos procedimentos de eletroestimulação (Tabela 1).

Nos indivíduos do grupo B, a força muscular dos quadríceps medida de forma objetiva com o dinamômetro

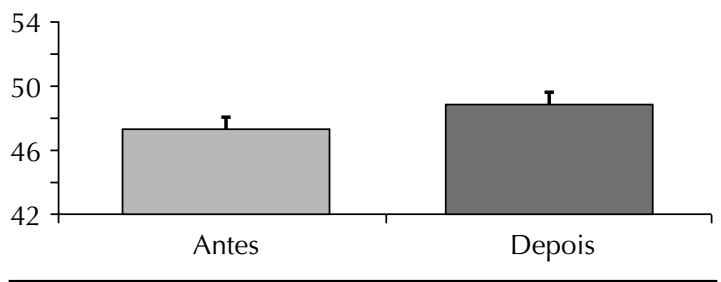

Figura 1. Força muscular de mulheres medida com dinamômetro (kgf) antes e após eletroestimulação (média \pm erropadrão). Maceió, AL, 2006. $\mathrm{N}=10$

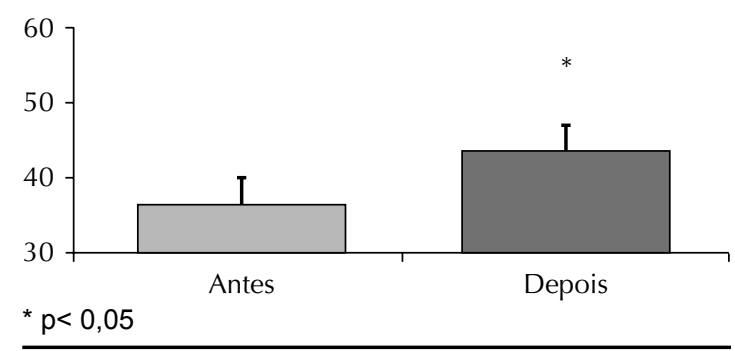

Figura 2. Força muscular de mulheres medida com dinamômetro (kgf) antes e após exercícios com carga (média \pm erro-padrão) Maceió, AL, 2006. N=10 teve média inicial igual a 36,2 kgf; IC95\%: 25,20; 47,19 e ao fim do treinamento, de $42,8 \mathrm{kgf}$; IC $95 \%$ : 33,$98 ; 51,61(\mathrm{p}<0,05)$, indicando aumento significante da força nos indivíduos submetidos a exercícios voluntários com carga (Figura 2). Quando a força muscular do quadríceps foi avaliada pela escala De Lorm, constatou-se aumento significante $(p=0,01)$ nos indivíduos do grupo $\mathrm{B}$, ao comparar as medidas finais $(9,3 \mathrm{kgf}$; IC $95 \%: 7,12 ; 11,48)$ às iniciais $(8 \mathrm{kgf}$; IC 95\%: 6,03;9,96). As medidas de cirtometria apresentaram alterações significantes ao longo das sessões de exercício (Tabela 2).

\section{DISCUSSÃO}

Os resultados do presente estudo mostraram que o exercício resistido de flexo-extensão dos joelhos durante três meses foi eficaz em promover melhora de massa e força muscular, medida de forma objetiva. Por outro lado, a aplicação de eletroestimulação por meio de um aparelho de uso doméstico, pelo mesmo período, não alcançou os mesmos resultados, pois não ocorreram aumentos significantes da massa e da

Tabela 1. Medidas da cirtometria acima dos joelhos direito (D) e esquerdo (E) em mulheres submetidas a sessões de eletroestimulação. Maceió, AL, 2006. N=10

\begin{tabular}{lcc}
\hline $\begin{array}{l}\text { Altura do } \\
\text { joelho }\end{array}$ & $\begin{array}{c}\text { Antes do exercício } \\
\text { Média (IC 95\%) }\end{array}$ & $\begin{array}{c}\text { Depois do exercício } \\
\text { Média (IC 95\%) }\end{array}$ \\
\hline $7 \mathrm{~cm}(\mathrm{D})$ & $37,7(36,51 ; 38,88)$ & $38,25(36,74 ; 39,75)$ \\
$7 \mathrm{~cm}(\mathrm{E})$ & $37,6(36,36 ; 38,83)$ & $38,7(37,19 ; 40,20)$ \\
$14 \mathrm{~cm}(\mathrm{D})$ & $43,05(41,56 ; 44,53)$ & $43,03(41,32 ; 44,73)$ \\
$14 \mathrm{~cm}(\mathrm{E})$ & $42,72(41,55 ; 43,89)$ & $43,18(40,42 ; 44,55)$ \\
$21 \mathrm{~cm}(\mathrm{D})$ & $48,6(47,29 ; 49,90)$ & $49,14(47,66 ; 50,61)$ \\
$21 \mathrm{~cm}(\mathrm{E})$ & $48,79(47,41 ; 49,98)$ & $49,3(47,65 ; 50,94)$ \\
$30 \mathrm{~cm}(\mathrm{D})$ & $54,95(53,09 ; 56,80)$ & $55,15(53,31 ; 56,99)$ \\
$30 \mathrm{~cm}(\mathrm{E})$ & $55,8(53,65 ; 57,94)$ & $56,1(53,90 ; 58,29)$ \\
\hline
\end{tabular}

Tabela 2. Medidas da cirtometria acima dos joelhos direito (D) e esquerdo (E) de mulheres após sessões de exercícios com carga. Maceió, AL, 2006. N=10

\begin{tabular}{lcc}
\hline $\begin{array}{l}\text { Altura do } \\
\text { joelho }\end{array}$ & $\begin{array}{c}\text { Antes do exercício } \\
\text { Média (IC 95\%) }\end{array}$ & $\begin{array}{c}\text { Depois do exercício } \\
\text { Média }(I C 95 \%)\end{array}$ \\
\hline $7 \mathrm{~cm}(\mathrm{D})$ & $34,35(31,58 ; 37,11)$ & $35,48^{*}(32,91 ; 38,04)$ \\
$7 \mathrm{~cm}(\mathrm{E})$ & $34,36(31,99 ; 36,72)$ & $35,42^{*}(33,07 ; 37,76)$ \\
$14 \mathrm{~cm}(\mathrm{D})$ & $39,2(36,62 ; 41,77)$ & $40,63^{*}(38,10 ; 43,15)$ \\
$14 \mathrm{~cm}(\mathrm{E})$ & $39,09(36,71 ; 41,48)$ & $40,6 * 37,76 ; 43,44)$ \\
$21 \mathrm{~cm}(\mathrm{D})$ & $44,75(47,29 ; 49,90)$ & $47,06^{*}(47,66 ; 50,61)$ \\
$21 \mathrm{~cm}(\mathrm{E})$ & $45,05(41,71 ; 48,39)$ & $47,26^{*}(43,72 ; 50,79)$ \\
$30 \mathrm{~cm}(\mathrm{D})$ & $51(47,40 ; 54,59)$ & $52,05^{*}(48,86 ; 55,23)$ \\
$30 \mathrm{~cm}(\mathrm{E})$ & $50,9(47,19 ; 54,60)$ & $52,87^{*}(49,53 ; 56,21)$ \\
\hline$* \mathrm{p}<0,05$ & &
\end{tabular}


força muscular quando medida de forma objetiva. No entanto, a comparação da força muscular medida de forma subjetiva antes e após cada um dos procedimentos, mostrou que ocorreu aumento da força em ambos os grupos. Assim, os aparelhos de eletroestimulação para ganho condicionamento físico divulgados comercialmente são menos eficientes do que a prática de exercício físico voluntário.

Esses resultados podem ser somados ao estudo de Angeli et al. ${ }^{a}$ Esses autores compararam a eletroestimulação abdominal com exercícios resistidos de flexão de tronco, mostrando que a ginástica passiva com aparelhos comerciais tem resultados mais modestos que os prometidos. Angeli et al ${ }^{\mathrm{a}}$ constataram que não houve aumento do consumo de oxigênio, não podendo, portanto aumentar o gasto calórico resultando na perda de peso.

Vengust et al $^{10}$ realizaram estudo comparando a eficiência de dois protocolos de tratamento para desordens patelares: exercícios com cargas altas e poucas repetições e eletroestimulação seletiva do vasto medial. Foi constatado que o treino voluntário resultou em maior aumento de força e funcionalidade que a estimulação elétrica. Em outro estudo, Grillo \& Simões ${ }^{2}$ também compararam eletroestimulação com contração muscular voluntária em dois grupos de dez mulheres treinadas em um total de 24 sessões. Ambos os grupos tiveram ganho de força, mas maior ganho foi observado no grupo que realizou atividade física voluntária. $\mathrm{O}$ fato do trabalho de Grillo \& Simões ${ }^{2}$ ter observado ganhos significantes no grupo que recebeu eletroestimulação, diferentemente do encontrado no presente estudo, pode ter decorrido de diferenças metodológicas como terem sido realizadas oito sessões a menos no presente estudo. Outra possível causa dessa diferença encontrada pode ser atribuída à característica da eletroestimulação aplicada, de $87 \mathrm{~Hz}$ e voltagem variável entre 25 a 132 10volts no presente estudo e no de Grillo \& Simões, ${ }^{2}$ pulsos de baixa freqüência $(0,538 \mathrm{~Hz})$ e voltagem de 56,8 volts.
Por outro lado, Silva et $\mathrm{al}^{9}$ mostraram ganhos semelhantes entre indivíduos submetidos à eletroestimulação ou exercício físico. Nesse estudo, os autores constataram aumentos significantes da espessura do tecido muscular por meio de ultra-sonografia em ambos os grupos, após 18 sessões. Isso pode ser atribuído ao tempo de eletroestimulação em cada sessão - 20 min - enquanto que no presente estudo as sessões duraram 15 min. Outro possível fator para a diferença das conclusões pode decorrer do método utilizado para avaliar a massa muscular. A ultra-sonografia empregada por Silva et $\mathrm{al}^{9}$ é mais precisa para avaliar a massa muscular que a cirtometria.

Da mesma forma que no presente estudo, Iwasaki et $\mathrm{al}^{3}$ encontraram ganhos de força muscular em indivíduos submetidos a eletroestimulação. Entretanto, eles aplicaram um programa híbrido, combinando eletroestimulação e exercício voluntário.

Todavia, ao utilizar método subjetivo para medir a força muscular, não foi encontrada diferença significante entre os indivíduos submetidos a eletroestimulação e aos submetidos a exercícios físicos voluntários com ganho de força com ambos os procedimentos. Pode-se supor que a medida pela escala De Lorm pode ser influenciada por aspectos motivacionais dos participantes da pesquisa, uma vez que se tratavam de estudantes universitárias com conhecimento teórico sobre o tema. No entanto esses resultados sugerem que a eletroestimulação poderia ter sido efetiva em aumentar a força muscular medida pelo dinamômetro caso o número de sessões fosse aumentado.

Em conclusão, o exercício físico resistido de flexoextensão dos joelhos foi efetivo em aumentar massa e força muscular quando aplicado com pelo menos 16 sessões durante dois meses. As 16 sessões de eletroestimulação com correntes de freqüência de pulsos de $87 \mathrm{~Hz}$ não foram suficientes para promover ganhos de massa e força muscular, quando medida de forma objetiva. 


\section{REFERÊNCIAS}

1. DeLorm TL, Watkins AL. Progressive resistance exercise. New York: Appleton-Century; 1951.

2. Grillo DE, Simões AC. Atividade física convencional (musculação) e aparelho eletroestimulador: Um estudo da contração muscular. Estimulação elétrica: mito ou verdade? Rev Mackenzie Educ Fis Esporte. 2003;2(2):31-43.

3. Iwasaki T, Shiba N, Matsuse H, Nago T, Umezu Y, Tagawa $Y$, et al. Improvement in knee extension strength through training by means of combined electrical stimulation and voluntary muscle contraction. Tohoku J Exp Med. 2006;209(1):33-40.

4. Kahn J. Principles and practice of electrotherapy. 4.ed. New York: Churchill Livingstone; 2000.

5. Aveiro MC, Granito RN, Navega MT, Driusso P, Oishi J. Influence of a physical training program on muscle strength, balance and gait velocity among woman with osteoporosis. Rev Bras Fisioter. 2006;10(4):441-8

6. Palma A. Atividade física, processo saúde-doença, e condições socioeconômicas: uma revisão da literatura. Rev Paul Educ Fis. 2000;14(1):97-106.

7. Piazzi AF, Ugrinowitsch C, Tricoli V. Mecanismos de adaptação ao treinamento com eletroestimulação transcutânea a altas e médias freqüências. J Exerc Sport Scie. [periódico online]. 2004[acesso em: 26/1/2006];3(2):1-12. Disponível em: http://ojs.c3sl. ufpr.br/ojs2/index.php/jess/article/view/2769/2282.

8. Powell KE, Blair SN. The public health burdens of sedentary living habits: theorical but realistic estimates. Med Sci Sports Exerc. 1994;26(7):851-6.

9. Silva JCE, Monteiro Neto LF, Cirillo F, Sauro EE, Colman SGL, Koeke P, et al. Efeitos da estimulação elétrica neuromuscular associada ou não a exercícios de contração muscular voluntária máxima. Fisioter Bras. 2005;6(3):186-91.

10. Strojnik V, Vengust R, Pavlovcic V. The effect of proprioceptive training on neuromuscular function in patients with patellar pain. Cel Mol Biol Lett. 2002;7(1):170-1. 